\title{
TWO REMARKS ON THE FRANKLIN SYSTEM
}

\author{
by P. WOJTASZCZYK
}

(Received 15th October 1984)

The aim of this note is to present two observations about the classical Franklin system. First we show that the Franklin system, when considered in the space generated by special atoms (as defined and studied by Soares de Souza in [11] and [12]) is an unconditional basis equivalent to the unit vector basis in $l_{1}$. In our second result we give conceptually simpler proofs and some extensions of the results of Bočkariov's [1] about the conjugate Franklin system.

For $n=2^{k}+l, l=0,1, \ldots, 2^{k-1}, k=0,1,2, \ldots$ we put $t_{n}=(2 l+1) 2^{-k-1}$. We also put $t_{-1}=0$ and $t_{1}=1$. The Franklin system $\left(F_{n}\right)_{n=-1}^{\infty}$ is an orthonormal system of piecewise linear functions on $[0,1]$ such that $F_{n}$ has nodes at $\left(t_{j}\right)_{j=-1}^{n}$. The fundamental result about the system $\left(F_{n}\right)_{n=-1}^{\infty}$ is the following proposition (see [5]).

Proposition 1. There exist constants $C$ and $q, 0<q<1$ such that for all $n$

$$
\left|F_{n}(t)\right| \leqq C \sqrt{n+2} q^{n\left|t-t_{n}\right|}
$$

and

$$
\left|F_{n}^{\prime}(t)\right| \leqq C(n+2)^{3 / 2} q^{n\left|t-t_{n}\right|} .
$$

Remark 1. Obviously for large $n$ we can replace $n+2$ by $n$ in these inequalities.

Now we define atomic Hardy spaces. For more detailed information the reader may consult [7]. We will restrict our attention to $1 / 2<p \leqq 1$ only. A $p$-atom is either a function constantly equal to 1 on $[0,1]$ or a function $a(t), 0 \leqq t \leqq 1$ such that

$$
\begin{gathered}
\int a(t) d t=0, \\
\text { supp } a(t) \subset I \quad \text { for some interval } I \subset[0,1], \\
\text { and } \quad|a(t)| \leqq|I|^{-1 / p} .
\end{gathered}
$$

The space $H_{p}[0,1]$ is defined as the space of all distributions $f$ such that $f=\sum_{j} \lambda_{j} a_{j}$ where $a_{j}$ 's are $p$-atoms and $\sum\left|\lambda_{j}\right|^{p}<\infty$. The quantity inf $\left\{\left(\sum\left|\lambda_{j}\right|^{p}\right)^{1 / p}: f=\sum \lambda_{j} a_{j}\right.$, each $a_{j}$ a $p$-atom, $\left.\sum\left|\lambda_{j}\right|^{p}<\infty\right\}$ is denoted by $\|f\|_{p}$. It was shown in [13], [14], [10] that $F_{n}$ is an unconditional basis in $H_{p}[0,1]$ for $1 / 2<p \leqq 1$. 
The following modification of 1-atoms has been considered in [11] and [12]. A function $a(t)$ on $[0,1]$ is called a special atom if it ither a constant 1 or

$$
\begin{aligned}
& \text { supp } a(t)=I \text { for some interval } I \subset[0,1] \text { and } \\
& a(t)=|I|^{-1} \text { on the left hand half of } I \text { and } \\
& a(t)=-|I|^{-1} \text { on the right hand half of } I .
\end{aligned}
$$

The space $B$ is defined as the space of functions $f$ such that $f=\sum \lambda_{j} a_{j}$ with $a_{j}$ 's special atoms and $\sum\left|\lambda_{j}\right|<\infty$. The norm is defined by $\|f\|_{B}=\inf \left\{\sum \mid \lambda_{j}: a_{j}\right.$ special atom, $\left.f=\sum \lambda_{j} a_{j}\right\}$. Our aim is to prove the following

Theorem 1. The function $f$ is in $B$ if and only if

$$
\sum_{n=-1}(n+2)^{-1 / 2}\left|\int_{0}^{1} f(t) F_{n}(t) d t\right|<\infty .
$$

The proof of this theorem is a modification of the proof of Theorem 2 of [14].

Lemma 1. There exists a constant $C$ such that

$$
\left\|F_{n}\right\|_{B} \leqq C(n+2)^{-1 / 2} \quad n=-1,0,1, \ldots
$$

The proof of this Lemma can be found in [3] and [13]. One has to note that in the course of proving the estimate for $\left\|F_{n}\right\|_{1}$ in [3] and [13] an explicit decomposition of $F_{n}$ into special atoms is obtained.

This Lemma immediately implies that whenever (3) holds $f$ is in $B$.

Lemma 2. Let a(t) be a special atom supported on the interval $I=(k, l)$ and let $\gamma(t, I)=$ $\min (|t-k|,|t-l|,|t-(k+l) / 2|)$. Then

$$
\left|\int_{0}^{1} a(t) F_{n}(t) d t\right| \leqq C|I|^{-1}(n+2)^{-1 / 2} q^{n \gamma\left(t_{n}, I\right)}
$$

for some constants $c>0$ and $q, 0<q<1$.

Proof. Let $h=\sum_{j=-1}^{n-1} b_{j} F_{j}$ be such that for $s<n$

$$
h\left(t_{s}\right)= \begin{cases}0 & \text { if } t_{s}<k \quad \text { or } t_{s}>1 \\ |I|^{-1} & \text { if } \quad k \leqq t_{s}<(k+l) / 2 \\ -|I|^{-1} & \text { if } \quad(k+l) / 2 \leqq t_{s} \leqq 1\end{cases}
$$

Then $\int_{0}^{1} a(t) F_{n}(t) d t=\int_{0}^{1}(a(t)-h(t)) F_{n}(t) d t$. Since $a(t)-h(t)$ is different from zero on at 
most three intervals around the points $k, l,(k+l) / 2$ and each interval is of the length at most $C(n+2)^{-1}$ we see that (4) follows from (1).

In order to prove Theorem 1 it is obviously enough to show that there is a constant $C$ such that for every special atom $a(t)$ we have $\sum(n+2)^{-1 / 2}\left|\int a F_{n}\right| \leqq C$. Let us fix $a(t)=|I|^{-1}\left(\chi_{1_{1}}-\chi_{1_{2}}\right)$ with $I_{1} \cup I_{2}=I, \quad I_{1} \cap I_{2}=\varnothing, \quad\left|I_{1}\right|=\left|I_{2}\right|$ and fix $N$ such that $2^{-N}<|I| \leqq 2^{-N+1}$. Let $A(t)=\int_{0}^{t} a(s) d s$. Using (2) we have

$$
\begin{aligned}
& \sum_{n=-1}^{2^{N}}\left|\int a(t) F_{n}(t) d t\right|(n+2)^{-1 / 2}=\sum_{n=-1}^{2^{N}}\left|\int A(t) F_{n}^{\prime}(t) d t\right|(n+2)^{-1 / 2} \\
& \quad \leqq C \sum_{n=-1}^{2^{N}} \int|A(t)| q^{n\left|t-t_{n}\right|} d t(n+2) \\
& \quad \leqq C \sum_{k=1}^{N} \sum_{2^{k-1}}^{2^{k}} \int|A(t)| q^{n\left|t-t_{n}\right|} d t 2^{k} \leqq C 2^{N} \int|A(t)| d t=\text { constant. }
\end{aligned}
$$

Using Lemma 2 we have

$$
\begin{aligned}
& \sum_{2^{N}}^{\infty}\left|\int a(t) F_{n}(t) d t\right|(n+2)^{-1 / 2}=\sum_{k=N}^{\infty} \sum_{n=2^{k}}^{2^{k+1}}\left|\int a(t) F_{n}(t) d t\right|(n+2)^{-1 / 2} \\
& \leqq C \sum_{k=N}^{\infty} \sum_{n=2^{k}}^{2^{k+1}} 2^{N}(n+2)^{-1} q^{n \gamma\left(t_{n}, l\right)} \\
& \leqq C \sum_{k=N} 2^{N} 2^{-k} \sum_{n=2^{k}}^{2^{k+1}} q^{n \gamma\left(t_{n}, I\right)} \\
& \leqq \text { constant. }
\end{aligned}
$$

So the Theorem is proved.

Remark 2. It is obvious that Theorem 1 holds also for orthonormal systems of the higher order splines defined in [4] and [6] (see also [14]).

Remark 3. Comparing Theorem 1 with the result of Ropela [9] one sees that $B$ is identical with some Besov space. This identification was shown in a different way in [11]. Those results suggest that possibility of "atomic decompositions" for other Besov spaces.

By $f$ we shall denote the trigonometric conjugate of a function $f \in L_{1}(T)$, i.e. a function such that $f+i f$ is a boundary value of an analytic function. The classical reference for the properties of the trigonometric conjugation is [15]. We shall consider the periodic Franklin system $f_{n}(t)$ on $T$. The definition is obvious (for details see [4] and [14]). For $f \in L_{1}(T)$ let $a_{k}(f)=\int f f_{k}$ and $b_{k}(f)=\int f f_{k}$. We shall use the following interpolation theorem which in much more general setting can be found in [8] Theorem 3.37. The proof in our case follows routinely from the classical Calderon-Zygmund decomposition. 
Proposition 2. Let $S$ be a sublinear operator defined on $L_{1}(T)$ with values in the space of measurable functions. If $\|S f\|_{L_{p}(T)} \leqq C\|f\|_{p}$ for some $C$ and some $p<1$ and $\|S f\|_{L_{q}(T)} \leqq C\|f\|_{L_{q}(T)}$ for some $q>1$, then $S$ is of weak type $1-1$, i.e.

$$
|\{t:|S f(t)| \geqq \lambda\}| \leqq B \lambda^{-1} \int|f|
$$

for some constant $B$.

It was shown in [10] and [14] that $\left(f_{n}\right)$ is an unconditional basis in $H_{p}(T)$ for $1 / 2<p \leqq 1$. Simce it is orthonormal, it is obviously an unconditional basis in $L_{2}(T)$. Also the trigonometric conjugation operator " $\sim$ " is the continuous operator on $H_{p}(T)$ and on $L_{2}(T)$ (see [7] and [15]). There is also a "formal identity" from $H_{p}(T)$ into $L_{p}(T)$ which is continuous. This "formal identity" is an extension of a map which sends an atom in $H_{p}$ to the same atom considered as an element of $L_{p}$. Using various compositions of these operators and Proposition 2 we obtain the following theorem.

Theorem 2. There exists a constant $C$ such that for all sequences $\mathscr{E}_{n}= \pm 1$ and for every $\lambda>0$ one has

$$
\begin{aligned}
& \left|\left\{t:\left|\sum \mathscr{E}_{n} a_{n}(f) f_{n}(t)\right|>\lambda\right\}\right| \leqq B \lambda^{-1} \int|f|, \\
& \left|\left\{t:\left|\sum \mathscr{E}_{n} a_{n}(f) \tilde{f}_{n}(t)\right|>\lambda\right\}\right| \leqq B \lambda^{-1} \int|f|, \\
& \left|\left\{t:\left|\sum \mathscr{E}_{n} b_{n}(f) f_{n}(t)\right|>\lambda\right\}\right| \leqq B \lambda^{-1} \int|f|, \\
& \left|\left\{t:\left|\sum \mathscr{E}_{n} b_{n}(f) \tilde{f}_{n}(t)\right|>\lambda\right\}\right| \leqq B \lambda^{-1} \int|f| .
\end{aligned}
$$

Inequality (6) was obtained by S. V. Bočkariov in [1] Theorem 10 and inequality (5) was also obtained by him in [2]. For an arbitrary unconditional basis $\left(G_{n}\right)$ in $H_{p}(T)$ we infer by the Khintchine inequality that the map

$$
f=\sum a_{n} G_{n} \mapsto\left(\sum\left|a_{n}\right|^{2}\left|G_{n}\right|^{2}\right)^{1 / 2}
$$

is bounded from $H_{p}(T)$ into $L_{p}(T), p<1$. This proves the following theorem.

Theorem 3. There exists a constant B such that

$$
\begin{aligned}
& \left\{t:\left(\sum\left|a_{n}(f)\right|^{2} f_{n}(t)^{2}\right)^{1 / 2}>\lambda\right\} \leqq B \lambda^{-1} \int|f| \\
& \left\{t:\left(\sum\left|a_{n}(f)\right|^{2} \widetilde{f}_{n}(t)^{2}\right)^{1 / 2}>\lambda\right\} \leqq B \lambda^{-1} \int|f| \\
& \left\{t:\left(\sum\left|b_{n}(f)\right|^{2} f_{n}(t)^{2}\right)^{1 / 2}>\lambda\right\} \leqq B \lambda^{-1} \int|f| \\
& \left\{t:\left(\sum\left|b_{n}(f)\right|^{2} \tilde{f}_{n}(t)^{2}\right)^{1 / 2}>\lambda\right\} \leqq B \lambda^{-1} \int|f| .
\end{aligned}
$$

As before (6a) was obtained first in [1] Theorem 9 and (5a) in [2]. 
Remark 4. Our argument is obviously much more general than we have stated. In particular one can replace $\left(f_{n}\right)$ by higher order spline systems and their derivatives and antiderivatives, i.e. all systems considered in Theorem $2^{\prime}$ of [14].

\section{REFERENCES}

1. S. V. BockarIov, Construction of polynomial bases in finite dimensional spaces of functions analytic in the unit disc, Proc. Steklov Inst. Math. 164 (1983), 49-74 (in Russian).

2. S. V. BoCKarIov, Existence of a basis in the space of functions analytic in the disc and some properties of the Franklin system, Mat. Sbornik (N.S.) 95 (137) (1974), 3-18 (in Russian).

3. Z. Ciesielski, The Franklin orthogonal system as unconditional basis in $\mathrm{ReH}_{1}$ and VMO, Functional Analysis and Approximation (ISNM 60, Birkhauser, 1981), 117-128.

4. Z. CIEsIELSKI, Bases and approximation by splines, Proc. Intl. Congress of Math. Vancouver $1974,47-51$.

5. Z. Ciesielski, Properties of the orthonormal Franklin system, Studia Math. 23 (1963), 141157. 302.

6. Z. Ciesielski, Constructive function theory and spline systems, Studia Math. 53 (1975), 277-

7. R. R. Coifman and G. Weiss, Extensions of Hardy spaces and their use in analysis, Bull. Amer. Math. Soc. 83 (1977), 569-645.

8. G. B. Folland and E. S. Stein, Hardy Spaces on Homogeneous Groups (Math. Notes 28, Princeton University Press).

9. S. Ropela, Spline bases in Besov spaces, Bull. Acad. Pol. Sci. 24 (1976), 319-325.

10. P. SJólin and J.-O. Stromberg, Basis properties of Hardy spaces, Arkiv för Math. 21 (1983), 111-125.

11. G. Soares de Souza and G. Sampson, A real characterisation of the predual of Bloch functions, J. London Math. Soc. 27 (1983), 367-376.

12. G. Soares De SouzA, Spaces formed by special atoms I, Rocky Mnts J. of Math. 14 (1984), 423-431.

13. P. Wojtaszczyk, The Franklin system is an unconditional basis in $H_{1}$, Arkiv för Math. 20 (1982), 293-300.

14. P. Wojtaszczyк, $\mathrm{H}_{p}$-spaces, $p \leqq 1$, and spline systems, Studia Math. 77 (1984), 289-320.

15. A. Zygmund, Trigonometric series (Cambridge University Press).

Institut of Mathematics

Polish ACademy of Sciences

Warsaw, Poland

and

St. John's College, Cambridge 